
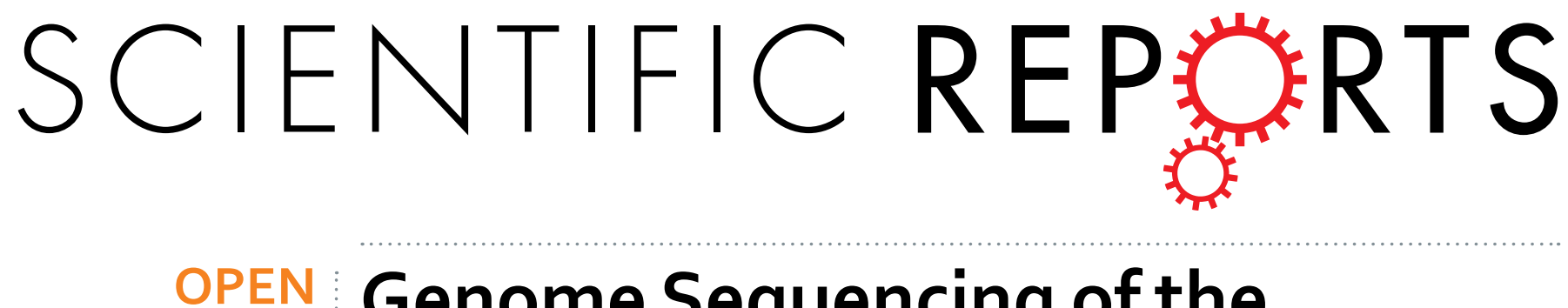

\title{
Genome Sequencing of the
} Pyruvate-producing Strain Candida glabrata CCTCC M202019 and

Received: 26 July 2016

Accepted: 21 September 2016

Published: 07 October 2016

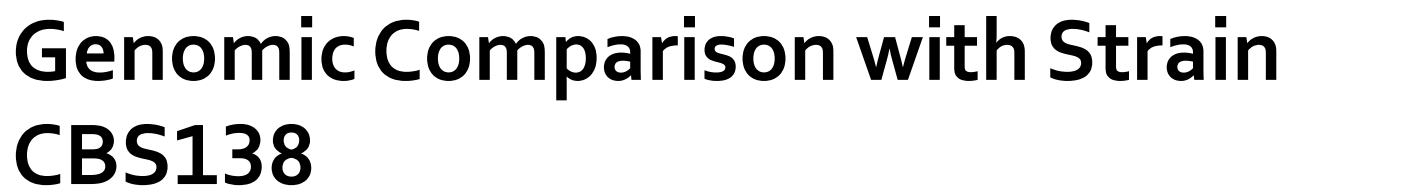

Nan $\mathrm{Xu}^{1,2}$, Chao Ye ${ }^{1,2}$, Xiulai Chen ${ }^{1,2}$, Jia Liu ${ }^{1,2}$, Liming Liu ${ }^{1,2}$ \& Jian Chen ${ }^{1,2}$

Candida glabrata CCTCC M202019 as an industrial yeast strain that is widely used to produce $\alpha$-oxocarboxylic acid. Strain M202019 has been proven to have a higher pyruvate-producing capacity than the reference strain CBS138. To characterize the genotype of the M202019 strain, we generated a draft sequence of its genome, which has a size of $12.1 \mathrm{Mbp}$ and a GC content of $38.47 \%$. Evidence accumulated during genome annotation suggests that strain M202019 has strong capacities for glucose transport and pyruvate biosynthesis, defects in pyruvate catabolism, as well as variations in genes involved in nutrient and dicarboxylic acid transport, oxidative phosphorylation, and other relevant aspects of carbon metabolism, which might promote pyruvate accumulation. In addition to differences in its central carbon metabolism, a genomic analysis revealed genetic differences in adhesion metabolism. Forty-nine adhesin-like proteins of strain M202019 were identified classified into seven subfamilies. Decreased amounts of adhesive proteins, and deletions or changes of low-complexity repeats and functional domains might lead to lower adhesion and reduced pathogenicity. Further virulence experiments validated the biological safety of strain M202019. Analysis of the C. glabrata CCTCC M202019 genome sequence provides useful insights into its genetic context, physical characteristics, and potential metabolic capacity.

Candida glabrata, previously designated Torulopsis glabrata, is a haploid yeast belonging to the Saccharomycetaceae family and the Candida genus. The C. glabrata CCTCC M202019 strain, originally screened from fertile soil niches, endured long-term and repeated domestication in laboratory broth ${ }^{1,2}$, and when used on an industrial scale, it can accumulate $84.2 \mathrm{~g} \cdot \mathrm{L}^{-1}$ pyruvate ${ }^{3}$. Pyruvate is an important chemical moiety that has been widely used in chemosynthesis, agrochemistry, dietary supplements, and pharmaceutical applications ${ }^{4,5}$. In addition, strain M202019 has the potential to produce $\alpha$-ketoglutarate ${ }^{6}$, fumarate ${ }^{7}$, acetoin ${ }^{8}$, malate ${ }^{9}$, and diacetyl ${ }^{10}$, as demonstrated by metabolic engineering experiments that increased the carbon flux from pyruvate nodes into these downstream chemicals. However, there is lack of a systematic understanding of its high pyruvate production capacity. To gain a comprehensive understanding of the genetic characteristics of strain M202019 for industrial-scale pyruvate production, and to improve its exploitation in industrial biotechnology, the genome of C. glabrata CCTCC M202019 needs to be sequenced, compared, and analyzed.

CBS138 was the first C. glabrata strain to have its whole genome sequenced ${ }^{11}$; C. glabrata is the second most frequent opportunistic yeast pathogen to be isolated from human feces and mucosa ${ }^{12}$. C. glabrata CBS138 can cause urogenital tract and bloodstream infections as a result of its own virulence-related traits, such as adherence, drug resistance, pigment production, acidic tolerance, and ace 2 mutations ${ }^{13}$. Among them, adherence is thought to be an extremely important virulence factor, and adherence is mediated by lectins that are encoded by epithelial adhesin (EPA) genes ${ }^{14}$. These EPA genes encode glycosylphosphatidylinositol (GPI)-anchored cell wall proteins

${ }^{1}$ State Key Laboratory of Food Science and Technology, Jiangnan University, 1800 Lihu Road, Wuxi, Jiangsu 214122, China. ${ }^{2}$ Key Laboratory of Industrial Biotechnology, Ministry of Education, School of Biotechnology, Jiangnan University, 1800 Lihu Road, Wuxi, Jiangsu 214122, China. Correspondence and requests for materials should be addressed to L.L. (email: mingll@jiangnan.edu.cn) 
that are covalently bound to cell wall glycoconjugates ${ }^{15}$. Its special cell wall architecture may help C. glabrata CBS138 to adhere to biotic and abiotic surfaces of mammalian cells. Both the niches and evolutionary history of strain CBS138 different from those of C. glabrata CCTCC M202019, which might lead to differences in the physiological and metabolic performance of these strains. The currently available C. glabrata CBS138 genome is a valuable tool for functional and comparative genomic research of C. glabrata.

In this study, we first showed that C. glabrata CCTCC M202019 has a higher pyruvate-producing capacity than C. glabrata CBS138. To determine key genotypic properties, the whole genome of C. glabrata CCTCC M202019 was de novo sequenced and mapped using high-throughput sequencing technology. The comprehensiveness and reliability of the function of M202019 genes were ensured by using various prediction and annotation tools. Then, a comparative genomic analysis was performed between the two strains. They shared highly similar genome structure and gene-order features, and genetic differences mainly appeared in pyruvate-related central carbon metabolism and toxic metabolism. Furthermore, the mechanism underlying the decreased virulence of C. glabrata CCTCC M202019 was resolved and validated. This study not only provides a genomic platform to understand the physiological and metabolic mechanisms of C. glabrata, but it also identifies potential genetic targets for the optimization of carbon metabolism and the reduction of toxicity.

\section{Results and Discussion}

Pyruvate fermentation in C. glabrata CCTCC M202019 and CBS138. It was reported that pyruvate production by C. glabrata CCTCC M202019 has reached an industrial level, and that other C. glabrata strains can accumulate pyruvate ${ }^{16}$. However, the pyruvate production capacity of C. glabrata CBS138 was never investigated. Therefore, pyruvate production by C. glabrata CCTCC M202019 and CBS138 was analyzed (Fig. 1). In commonly used media, i.e., synthetic complete (SC), yeast extract peptone dextrose (YPD), and basal fermentation (BF) media, strain CBS138 grew slightly faster than strain M202019, especially in YPD medium (Fig. 1 and Supplementary Table S1a-c). An obvious growth difference was observed in an optimized fermentation (OF) medium. After culturing for $48 \mathrm{~h}$, strain M202019 reached $8.09 \mathrm{~g} \cdot \mathrm{L}^{-1}$ of biomass and just entered into stationary phase, whereas the biomass of strain CBS138 only reached $3.44 \mathrm{~g} \cdot \mathrm{L}^{-1}$ (Supplementary Table S1d), and it entered into stationary phase after approximately $30 \mathrm{~h}$. Glucose consumption of the two strains was similar to their cell growth curves in these media (Fig. 1 and Supplementary Table S1a-c). Glucose was consumed slightly faster by strain CBS138, compared with strain M202019, especially in YPD medium. In contrast, in OF medium, the glucose consumption rates of strains M202019 and CBS138 were $2.03 \mathrm{~g} \cdot \mathrm{L}^{-1} \cdot \mathrm{h}^{-1}$ and $1.74 \mathrm{~g} \cdot \mathrm{L}^{-1} \cdot \mathrm{h}^{-1}$, respectively. Additionally, pyruvate production by C. glabrata CCTCC M202019 was 39\%, 61\%, 66\%, and 95\% higher than that of strain CBD138 in SC, YPD, BF, and OF media, respectively. The most significantly improvement in OF could be reflected by the fact that $42.32 \mathrm{~g} \cdot \mathrm{L}^{-1}$ pyruvate accumulated in strain M202019, while strain CBS138 only accumulated $18.7 \mathrm{~g} \cdot \mathrm{L}^{-1}$ pyruvate at $48 \mathrm{~h}$. Meanwhile, pyruvate yields and productivity by strain M202019 were $0.21 \mathrm{~g} \cdot \mathrm{g}^{-1}$ and $0.41 \mathrm{~g} \cdot \mathrm{L}^{-1} \cdot \mathrm{h}^{-1}$ higher, respectively, than those in strain CBS138 (Supplementary Table S1d). In general, although the two strains accumulated pyruvate, either in commonly used media or in an optimized medium, the pyruvate production, yield, and productivity of strain M202019 were higher than those of strain CBS138, especially in the optimized medium. The greater pyruvate productivity exhibited by C. glabrata CCTCC M202019 might have evolved selectively during laboratory mutagenesis. To extensively analyze phenotypical difference and explore candidates for improving the pyruvate production capacity, the whole genome of the pyruvate-producing M202019 strain was sequenced and compared with the genome of strain CBS138.

Genome sequencing and genome characteristics of C.glabrata CCTCC M202019. The C. glabrata CCTCC M202019 genome was sequenced using the Illumina Solexa HiSeq 2000 platform. The $12.1 \mathrm{Mbp}$ genome sequence, with an average $\mathrm{G}+\mathrm{C}$ content of $38.47 \%$, was acquired from a 150 bp pair-end library with 208 -fold coverage, a 300 bp pair-end library with 165-fold coverage, and one mate-pair library with 106 fold coverage (Supplementary Fig. S1). High-quality reads were assembled into 111 contigs and 74 scaffolds. The N50 sizes of the contigs and scaffolds were 659,495 and 775,409, respectively (Table 1). A total of 5345 genes were predicted, 293 of which were generated by alternative splicing. Subsequently, gene function was annotated, including 4788 genes that were classified by Gene Ontology and 3088 genes that were classified by EuKaryotic Orthologous Groups (KOG) (Supplementary Dataset S1). In addition, 191 tRNA and six rRNA genes were identified (Table 1). The total repeat sequences occupied $1.15 \%$ of the genome (Supplementary Table S2), which included 16 short interspersed elements (1673 bp), 11 unclassified interspersed elements (11,449bp), 2644 simple repeats $(121,642 \mathrm{bp}), 365$ low-complexity repeats $(17,220 \mathrm{bp})$, and two long terminal repeats (776 bp).

Overview of comparative genomics between C. glabrata CCTCC M202019 and CBS138. Comparative genomics of C. glabrata CCTCC M202019 and CBS138 was investigated using the GO and KOG functional annotations, a homologous comparison of gene sequences, and the detection of single nucleotide polymorphisms (SNPs). First, 2792 GO terms were shared by the two strains (Supplementary Dataset S1), while strains M202019 and CBS138 had 37 and 24 unique GO terms, respectively (Supplementary Table S3). The two strains shared 2301 KOG categories (Supplementary Dataset S1), while 188 and 63 unique KOG categories were found in the M202019 and CBS138 strains, respectively (Supplementary Fig. S2). Additionally, the same genes of strains M202019 and CBS138 occupied 94.6\% and 95.1\% (Supplementary Dataset S1), respectively, of their genomes, which accounted for the majority of the core metabolic pathways. Other genes were ranked by their identities (Supplementary Table S4). The total of 275 and 159 genes exhibited greater than $90 \%$ identity in the M202019 and CBS138 strains, respectively. Sixteen and 43 genes exhibited less than 90\% identity in the M202019 and CBS138 strains. Noticeably, $74.3 \%$ of them were predicted to function in cell wall adhesion. Twelve unique genes in strain CBS138 belonged to adhesion clusters and genetic information process. The unique gene in strain M202019 encoded a ribulose-1,5-bisphosphate carboxylase/oxygenase subunit. A total of 205 SNPs were 

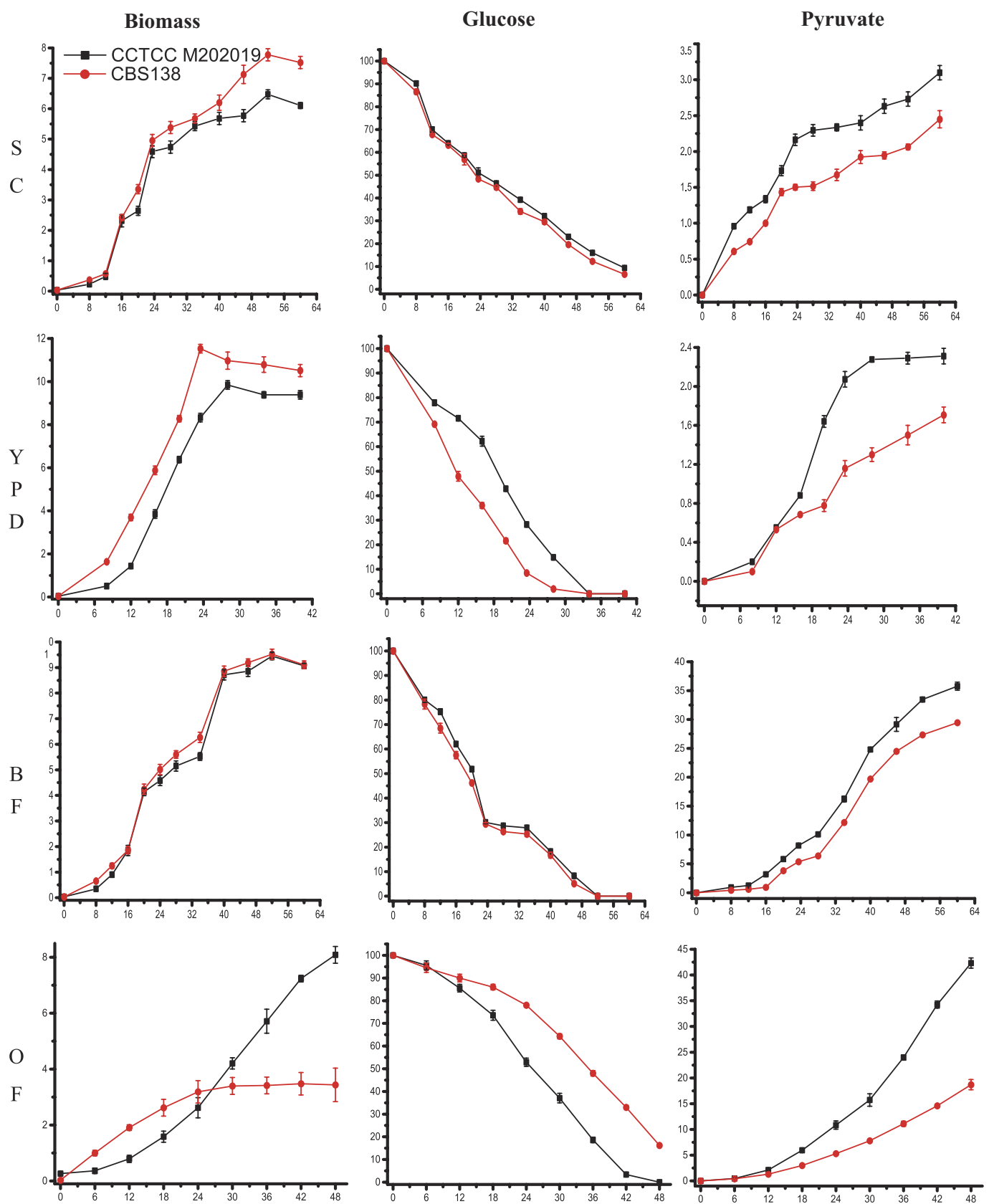

Figure 1. Pyruvate fermentation process of C. glabrata CCTCC M202019 and CBS138. Synthetic complete medium (SC), yeast extract peptone dextrose medium (YPD), basal fermentation medium (BF), and optimized fermentation medium $(\mathrm{OF})$; units, $\mathrm{g} \cdot \mathrm{L}^{-1}$.

preliminarily identified (Supplementary Table S5), 61 of which were scattered in the regulatory regions of 25 genes (Supplementary Table S7), while the rest were located in the open reading frames of 51 genes (Supplementary Table S6). Among the 51 genes, 35 belonged to verified or hypothetical proteins, and 16 were pseudogenes. The 35 verified or hypothetical proteins functioned in cell adhesion $(22.9 \%)$, central carbon metabolism (22.9\%), and non-metabolic cellular processes (45.7\%), and 31 of them acquired sense mutations, while the other four contained nonsense mutations. Additionally, 13 of the 16 pseudogenes were predicted to be related to cell adhesion. Overall, the comparative genomics analysis suggests a high similarity of these two closely related strains, and the limited number of genetic mutations is likely related to certain phenotypic differences.

Genomic characteristics accounting fhor high pyruvate productivity in C. glabrata CCTCC

M202019. The main factors influencing pyruvate metabolism in C. glabrata include pyruvate biosynthesis from glucose via glycolysis, the pentose phosphate shunt, and methylglyoxal degradation pathways, and an efficient uptake and transport system that is important for pyruvate production ${ }^{17}$. Additionally, the high pyruvate accumulation could result from the auxotrophies of four vitamins, which function as the cofactors of key enzymes 


\begin{tabular}{|l|c|}
\hline General features & 12.1 \\
\hline Genome size (Mb) & 38.47 \\
\hline GC content (\%) & 111 \\
\hline Number of contigs & 659,495 \\
\hline Contig N50 & 74 \\
\hline Number of scaffolds & 775,409 \\
\hline Scaffold N50 & 1.15 \\
\hline Length of classified repeats (\%) & 5,345 \\
\hline Properties of gene annotation & 191 \\
\hline Number of protein-coding sequences & 6 \\
\hline Number of tRNA genes & 961 \\
\hline Number of rRNA genes & 4,788 \\
\hline Number of genes with EC assignment & 3,088 \\
\hline Number of genes with GO assignment & \\
\hline Number of genes with KOG assignment &
\end{tabular}

Table 1. Genome characteristics of C. glabrata CCTCC M202019.

\begin{tabular}{|l|l|l|}
\hline Subsystems & Function & Genes \\
\hline \multirow{4}{*}{ Transport } & glucose & HXT3 and HXT4/6/7 \\
\cline { 2 - 3 } & niacin & TNR1 and TRN2 \\
\cline { 2 - 3 } & acetic acid & CAGL0M03465g \\
\cline { 2 - 3 } & dicarboxylic acids & CAGL0J04114g \\
\hline \multirow{5}{*}{ Oxidative phosphorylation } & cytochrome C oxidase & $\begin{array}{l}\text { COX1, COX2, COX7A, } \\
\text { COX7C, and COX17 }\end{array}$ \\
\cline { 2 - 3 } & cytochrome C reductase & CYTB and OCR10 \\
\cline { 2 - 3 } & F-type ATPase & atp8, $\mathrm{j}$, and $\mathrm{k}$ \\
\cline { 2 - 3 } & ETC complex IV & PET309 \\
\hline \multirow{2}{*}{ Downstream metabolism } & acetyl-CoA hydrolase & ACH1 and GLN1 \\
\cline { 2 - 3 } & glutamate synthase & GLT1 \\
\hline
\end{tabular}

Table 2. Genetic differences related to pyruvate production in C. glabrata.

in pyruvate metabolic pathways that weaken pyruvate degradation ${ }^{18}$. Furthermore, pyruvate production can be enhanced by altering the transmission of reducing power, as well as energy production, by engineering oxidative phosphorylation to strengthen NADH oxidation ${ }^{19}$ and decrease ATP levels ${ }^{20}$. Finally, some other by-products, such as tricarboxylic acid metabolites, could compete with pyruvate for carbon flux. Compared to the genome of C. glabrata CBS138, the genome of strain M202019 had some changes in central carbon metabolism (Table 2), including transport proteins such as glucose transporters, niacin transporters, an acetic acid transporter, and a transporter that transfers dicarboxylic acid transporter between mitochondria and the cytoplasm. Other genes were involved in oxidative phosphorylation, including cytochrome $\mathrm{C}$ oxidase, cytochrome $\mathrm{C}$ reductase, an F-type ATPase, and assembly and biogenesis proteins of electron transport chain complex IV. Other genes that are relevant to metabolism include acetyl-CoA hydrolase, and involved in acetate formation and glutamate synthase as joints of carbon and nitrogen metabolism. The aforementioned changes in central carbon metabolism genes might be associated with higher levels of pyruvate production by C. glabrata CCTCC M202019.

Identification and classification of adhesin proteins in C. glabrata CCTCC M202019. In addition to those involved pyruvate-related central carbon metabolism, many genetic differences were also identified in adhesion metabolism. In C. glabrata CBS 138, 62 adhesion proteins were predicted by FungalRV using a threshold of 0.511 including 20 adhesins identified in the proteome ${ }^{21}$. FaaPred also successfully predicted adhesin-like proteins and supplement-related annotations such as CAGL0I10246g, CAGL0J02508g, and CAGL0I11011g in C. glabrata CBS 138. The intersection of results from two programs were considered to reduce false positives. Using the protein sequences of C. glabrata CCTCC M202019, 75 and 78 fungal adhesins and adhesin-like proteins were predicted by FungalRV and FaaPred, respectively. Among the shared 64 proteins, 49 possessed typical adhesion protein structures (Fig. 2a), including a GPI-anchor, low-complexity serine (S)/threonine (T) repeats, conserved (V)SHITT/TTVVT amino acid motifs, hyphally-regulated cell wall proteins (Hyphal_reg_CWP), PA14 and its similar structural domain, and flocculin repeats (Supplementary Table S8). These 49 proteins are thought to be putative adhesins of strain M202019, and they were divided into seven subtypes according to their amino-terminal structures (Fig. 2b), which is consistent with adhesin protein classification for C. glabrata $\mathrm{CBS}_{138}{ }^{22}$. The largest subgroup (I) contained 14 proteins, and its nearest homology subgroup (II) comprised six proteins. Both subgroups had conserved PA14 and PA14-like domains, wherein g4656, g835, and g3240 also had Hyphal_reg_CWP and flocculin repeats. Three adhesins in subset IV had a GPI anchor. Almost all of the 
$\mathbf{a}$

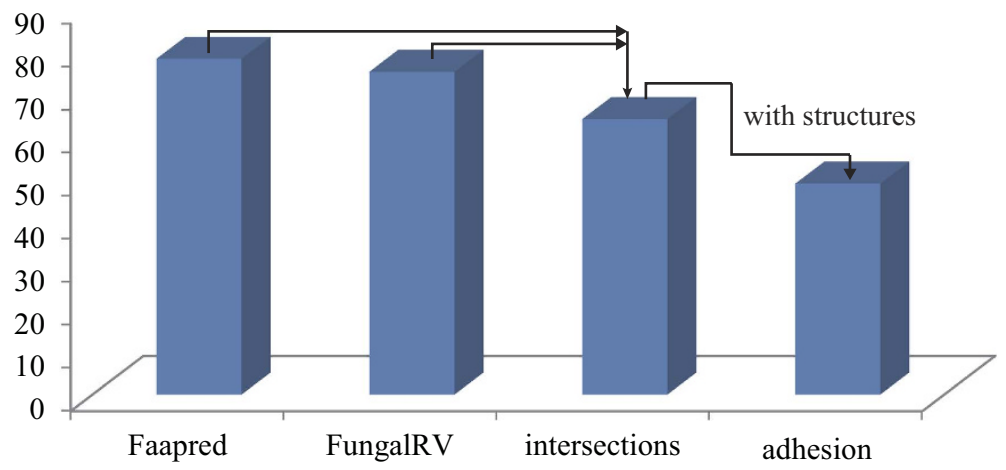

b

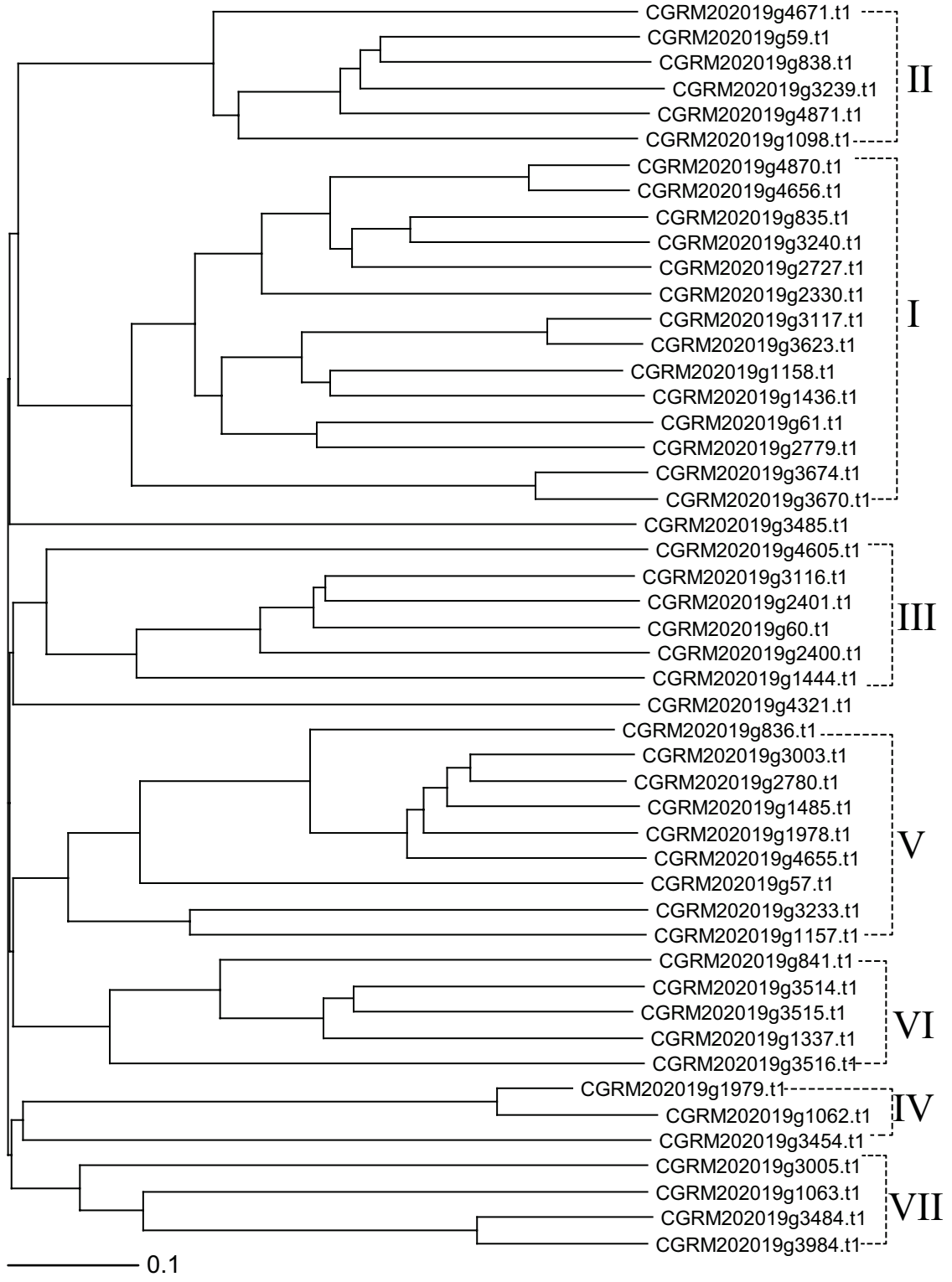

Figure 2. Identification and classification of adhesin-like proteins in C. glabrata CCTCC M202019 (a) Identification of adhesin-like proteins (b) Classification of adhesin-like proteins.

adhesins in subgroups V and VI contained Hyphal_reg_CWP protein structures, which indicated the functional similarities of the two subgroups. Non-characteristic domains were found in subgroups III and VII, while all 


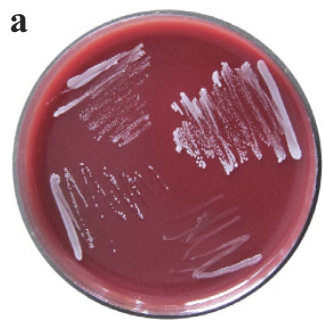

b

\begin{tabular}{cc}
\hline Strains & $\begin{array}{c}\text { Protease } \\
\text { activity }\end{array}$ \\
\hline C. glabrata CBS138 & 3.05 \\
C. glabrata CCTCC & 1.46 \\
M202019 & \\
C. albicans SC5314 & 3.45 \\
S. cerevisiae BY4742 & 0.62 \\
\hline
\end{tabular}

c
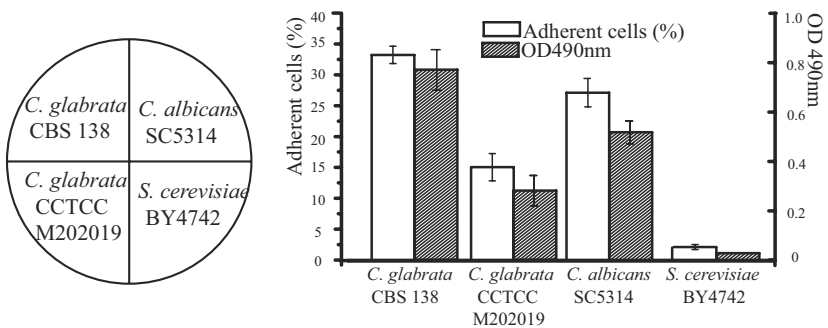

Figure 3. Virulence and adhesion testing of C. albicans SC5314, S. cerevisiae BY4742, and C. glabrata CCTCC M202019 and CBS138 (a) Cell growth on Columbia agar base; (b) protease activity; (c) adhesion to endothelial cells and 96-microwell plates.

seven families possessed low-complexity repeats. Additionally, the conserved repeats SHITT and TTVVT were distributed in most subgroups, including subgroups I, III, IV, and V.

Structural variations of adhesins in C. glabrata. The comparative genomics analysis suggested that only six adhesins were shared by the two strains, and the remaining 54 hypothetical proteins and 12 pseudogenes in C. glabrata CBS138 all differed. For 47 of the 54 adhesins, variations located in low-complexity repeats were rich in serine and threonine. The mutations and deletions in the aforementioned tandem repeats could cause the amino-terminal effector domains of these adhesins to become buried in the cell wall ${ }^{23}$. Compared with low-complexity repeats, other structural domains seemed to exhibit lower variation frequencies, including the PA14 domains of CAGL0E06688g, the flocculin repeats of CAGL0I07293g and CAGL0I00220g, Hyphal_reg_ CWP of CAGL0J02530g and CAGL0F09273g, and the GPI-anchor regions of CAGL0L09911g, CAGL0L00157g, and CAGL0H10626g. These functional domains are reported to be individually instrumental in adhesion to human tissue ${ }^{14,24,25}$. For example, PA14 domains, specifically the ligand-binding domains of EPA proteins, were found in bacterial toxins, glucosidases and adhesins. Deletion of C. glabrata genes with PA14 domains such as CAGL0I10098g reduce its adherence to endothelial cells ${ }^{26}$. Flocculin repeats involved in flocculation and cell adhesion, and yeast strains with a larger number of repeats in the FLO1(YAR050W) gene exhibit better adhesion than those with a smaller number of repeats ${ }^{27}$. Therefore, these changes in the functional domains of adhesins were proposed to reduce the adherence of C. glabrata. In addition, of the 12 changed pseudogenes that were associated with cell aggregation, the distribution of the mutation sites was comparatively dispersed in five pseudogenes, but relatively concentrated in the other seven pseudogenes (Supplementary Table S9). These differences in adhesin-encoding genes might reduce cell adherence and virulence.

Evaluation of the biological safety of C. glabrata CCTCC M202019. The virulence and adherence of C. glabrata CCTCC M202019 was tested and compared to validate the aforementioned hypothesis. The pathogenicity of strains M202019 and CBS138 could be reflected in their ability to grow on Columbia agar base; thus, the growth of Candida albicans SC5314, Saccharomyces cerevisiae, and C. glabrata CCTCC M202019 and CBS138 was tested on this medium. The highly virulent $C$. albicans exhibited the highest growth rate, followed by strain CBS138 while strain M202019 and S. cerevisiae grew slowly (Fig. 3a). The virulence of such strains is generally proportional to their protease producing capacity. Among these four strains, C. albicans SC5314 and C. glabrata CBS138 exhibited a high protease-producing capacity, with protease activities of 3.45 and 3.05, respectively (Fig. 3b). Strain M202019 was a less virulent strain, with a protease activity of 1.46, while S. cerevisiae colonies did not generate transparent halos. Thus, based on its growth rate and protease-producing capacity, C. glabrata CCTCC M202019 appears to be less virulent and, hence, safer than C. glabrata CBS138.

The toxicity and pathogenicity of Candida mainly results from a membrane structure that enables adherence to the surfaces of biological or non-biological materials ${ }^{28}$. Here, the adhesion of C. albicans SC5314, S. cerevisiae, and C. glabrata CCTCC M202019 and CBS138 to endothelial cells and the inner wall of pipes was investigated. The adhesion rate of strain CBS138 to endothelial cells was 33\%, and the next most adhesive strain was the highly virulent C. albicans. The adhesion rate of strain M202019 (15\%) was less than half of that of C. glabrata CBS138. Almost no endothelial cells exhibited adherent S. cerevisiae, which is non-virulent. The adhesion of the four experimental strains on 96-microwell plates is also shown in Fig. 3c. C. glabrata CBS138 had the strongest adhesion, followed by C. albicans SC5314. Compared with C. glabrata CBS138, the adhesion of strain M202019 on 96 -microwell plates was $63.6 \%$ lower. The absorbance value of adherent cells was close to 0 for S. cerevisiae. We conclude that the adhesive capacity of strain M202019 was only 40-50\% of that of C. glabrata CBS138 on either 
biological (endothelial cells) or non-biological (96-microwell plates) surfaces. The lower adhesive ability of the pyruvate-producing strain C. glabrata CCTCC M202019 was predicted by the genomics analysis, possibly due to the decreased amounts of adhesive proteins, and deletion or mutations in low-complexity repeats in this strain.

\section{Methods}

Genome sequencing and annotation. C. glabrata CCTCC M202019 was preserved in the China Center for Type Culture Collection (Wuhan, Hubei, China). Genomic DNA was extracted from fresh cells during logarithmic growth phase, and DNA was sequenced using the Illumina (San Diego, CA, USA) Solexa HiSeq 2000 platform. Three libraries were constructed. Two paired-end libraries were prepared with $150 \mathrm{bp}$ and $300 \mathrm{bp}$ fragments. These libraries generated 22,645,461 reads. A mate-pair library that was prepared with $6 \mathrm{~kb}$ fragments yielded $6,492,803$ reads. Approximately $5.8 \mathrm{~Gb}$ were qualified using the NGS QC Toolkit ${ }^{29}$ and constructed into contigs using Velvet ${ }^{30}$ and scaffolds using SSPACE ${ }^{31}$. Gaps were modified and closed using GapFiller ${ }^{32}$.

Repeat sequences in the assembled contigs were marked and removed using RepeatModeler and RepeatMasker ${ }^{33}$. Expressed sequence tags and protein sequences from phylogenetically related microorganisms were compared to C. glabrata CCTCC M202019 contigs using Exonerate ${ }^{34}$. Sequence matches were used for gene prediction with AUGUSTUS ${ }^{35}$. Functions of putative genes were annotated using the Kyoto Encyclopedia of Genes and Genomes Automatic Annotation Server (KOG) ${ }^{36,37}$ and Gene Ontology annotation ${ }^{38}$ tools. In addition, tRNA were identified using tRNAscan-SE ${ }^{39}$ and rRNA genes using RNAmmer ${ }^{40}$.

Comparative genomics. A comparative genomic analysis between C. glabrata CCTCC M202019 and CBS 138 was performed by homology comparison of genes and detection of single nucleotide polymorphisms. Genes in the two strains were compared with the local nucleotide Basic Local Alignment Search Tool (BLASTN) ${ }^{41}$, and the alignment results were approximately divided into four categories, i.e., exactly the same, high homology with minimum standards of $90 \%$ identity and $80 \%$ length coverage, non-homologous relations with less than $90 \%$ identity or less than $80 \%$ length coverage, and completely non-matched genes.

Raw reads that were generated by the two pair-end libraries were filtered by the NGS QC Toolkit(available at http://www.nipgr.res.in/ngsqctoolkit.html), and then mapped to the 13 chromosomes of C. glabrata CBS138 using Bowtie $2^{42}$. The mapping files in the sequence alignment/map (SAM) format were converted, and the sorted and indexed binary versions of SAM files were generated using SAMtools ${ }^{43}$. Polymerase chain reaction duplicates that were generated during library construction were removed by Picard tools (http://picard.sourceforge.net). Since mapping errors occur frequently around known indels, the RealignerTargetCreator and IndelRealigner programs in the Genome Analysis Toolkit (GATK) ${ }^{44}$ software were used to reduce false-positives during SNP prediction. Finally, variant calling was recalibrated from the consistent results of the GATK and SAMtools. The SNPs in the Visual Component Framework format were annotated as a tab delimited file using the SNP Data Analysis $\mathrm{Tool}^{45}$, and they were located in specific genes using Artemis ${ }^{46}$ tools.

Fermentation experiments. Four media were used to detect pyruvate production: SC medium (per liter, $100 \mathrm{~g}$ glucose and $6.7 \mathrm{~g}$ yeast nitrogen base without amino acids), YPD medium (per liter, $100 \mathrm{~g}$ glucose, $20 \mathrm{~g}$ peptone, and $10 \mathrm{~g}$ yeast extracts), $\mathrm{BF}$ medium ${ }^{16}$, and $\mathrm{OF}$ medium ${ }^{47}$. After sterilization at $115^{\circ} \mathrm{C}$ for $10 \mathrm{~min}, 40 \mathrm{~g} \cdot \mathrm{L}^{-1}$ $\mathrm{CaCO}_{3}$ was added to buffer the $\mathrm{pH}$.

C. glabrata CCTCC M202019 and CBS138 from agar slants ${ }^{48}$ were cultivated respectively in $50 \mathrm{~mL}$ and $500 \mathrm{~mL}$ of seed flask medium ${ }^{48}$ at $30^{\circ} \mathrm{C}$, with shaking at $200 \mathrm{rpm}$. Five milliliters of seed culture in the logarithmic growth phase was transferred into $50 \mathrm{~mL}$ of fermentation medium in $500 \mathrm{~mL}$ flasks. The fermentation broth was adjusted to an initial $5.5 \mathrm{pH}$, and the strains were cultivated for $48 \mathrm{~h}$. The cell concentration ${ }^{49}$, and glucose ${ }^{19}$ and pyruvate $^{1}$ contents were measured during the fermentation process.

Virulence testing. Strains and cells. To compare the virulence of C. glabrata CCTCC M202019 and CBS138, the high virulent C. albicans SC5314 strain and the non-virulent S. cerevisiae BY4742 strain were chosen as positive and negative controls, respectively. Vascular endothelial cells were suspended by digestion with trypsin and counted using Trypan blue staining; greater than $95 \%$ of the cells were alive.

Media. Enzyme-producing medium contained (per liter) $20 \mathrm{~g}$ glucose, $10 \mathrm{~g}$ yeast extract, $20 \mathrm{~g}$ peptone, and $1 \mathrm{~g}$ $\mathrm{KH}_{2} \mathrm{PO}_{4}$, with an initial $\mathrm{pH}$ of 4.5. YGM2 milk medium contained (per liter) $20 \mathrm{~g}$ glucose, $10 \mathrm{~g}$ yeast extract, $1 \mathrm{~g}$ $\mathrm{KH}_{2} \mathrm{PO}_{4}$, and $15 \mathrm{~g}$ agar, with an initial pH of 4.5 . After sterilization at $115^{\circ} \mathrm{C}$ for $15 \mathrm{~min}, 1 \%$ (v/v) skimmed milk was added. Sabouraud's dextrose medium contained (per liter) $40 \mathrm{~g}$ glucose and $10 \mathrm{~g}$ peptone.

Activation. Four experimental strains were activated using the enzyme-producing medium and then transferred to Columbia agar base. Both strains were cultivated at $37^{\circ} \mathrm{C}$ for $24 \mathrm{~h}$ to recover their biological activities.

Protease activity. Single colonies of experimental strains that were cultivated on Columbia agar base and preserved at $4^{\circ} \mathrm{C}$ were spotted onto YGM2 milk medium. After cultivation for $72 \mathrm{~h}$ at $37^{\circ} \mathrm{C}$, the diameter of the colonies and the surrounding transparent zones were measured. The protease activity (A) was equal to the diameter of the transparent zone divided by the colony diameter ${ }^{50}$.

Adherence assay. The concentrations of experimental strains and vascular endothelial cells were adjusted to $2 \times 10^{6} \mathrm{~mL}^{-1}$ and $10^{5} \mathrm{~mL}^{-1}$, respectively, using Hanks' balanced salt solution. The two were mixed in equal volumes $(1 \mathrm{~mL})$ and then incubated at $37^{\circ} \mathrm{C}$ in $5 \% \mathrm{CO}_{2}$ for $24 \mathrm{~h}$. After staining and observation, the number of yeast adhering to more than three endothelial cells were counted, and the percentage of adherent cells calculated ${ }^{51}$. 
Single colonies of the experimental strains were incubated in fresh Sabouraud's dextrose medium at $37^{\circ} \mathrm{C}$, with shaking at $200 \mathrm{rpm}$. After reaching the late stage of growth, the number of cells was adjusted to $2 \times 10^{6} \mathrm{~mL}^{-1}$, and $100 \mu \mathrm{L}$ of a cell suspension was cultured for $4 \mathrm{~h}$ at $37^{\circ} \mathrm{C}$, with shaking $200 \mathrm{rpm}$. After discarding the medium and washing away non-adherent cells, $60 \mu \mathrm{L}$ of 2,3-Bis-(2-methoxy-4-nitro-5-sulfophenyl)-2H-tetrazolium5 -carboxanilide was immediately added, and the 96-microwell plates were preserved in dark for $2 \mathrm{~h}$. Adhesion capacity was determined by measuring the optical density at $490 \mathrm{~nm}^{52}$.

In silico analysis of adhesin-like proteins in C. glabrata CCTCC M202019. The epithelial adhesin genes in C. glabrata CBS138 were collected from the Candida Genome Database (http://www.candidagenome. $\mathrm{org} /$ ), and the FLO genes in S. cerevisiae mediating yeast flocculation and adherence to abiotic surfaces ${ }^{25,53}$ were used as the local BLASTN database. Additionally, proteins containing Pfam PF10528 domains in C. glabrata CCTCC M202019 were searched by the hmmscan command with an e-value less than $1 \mathrm{e}-05$. The union genes were further submitted to the FungalRV ${ }^{21}$ and FaaPred ${ }^{54}$, and the two intersection was detected as adhesin proteins. These proteins' domains were predicted with the SMART ${ }^{55}$ and PredGPI algorithms ${ }^{56}$. A phylogenetic tree of the adhesin-like proteins in C. glabrata CCTCC M202019 was constructed based on the clustering of the 1000 nucleotides from the $5^{\prime}$ end of EPA genes using BioEdit ${ }^{57}$.

\section{Conclusion}

The pyruvate-producing strain C. glabrata CCTCC M202019 was sequenced and compared with the genome of C. glabrata CBS 138. Although the two strains generally had high similarity, genetic differences were identified mainly in central carbon metabolism and adhesion metabolism. Fermentation experiments and virulence testing supported the phenotypes of higher pyruvate producing and lower toxicity deduced from genotypes of C. glabrata CCTCC M202019. The study could be used for targeted improvements in pyruvate yield in C. glabrata and rationally engineering other industrial microorganism to produce pyruvate. Strategies for lowering the virulence of C. glabrata CBS 138 were suggested such as decreasing the number of low-complexity repeats of adhesins and mutating their functional domains. The data obtained in this study will facilitate the optimization of industrial bioprocess and further exploration of the pathogenicity of C. glabrata.

\section{References}

1. Li, Y., Chen, J., Lun, S. Y. \& Rui, X. S. Efficient pyruvate production by a multi-vitamin auxotroph of Torulopsis glabrata: key role and optimization of vitamin levels. Appl. Microbiol. Biotechnol. 55, 680-685 (2001).

2. Liu, L. M., Li, Y., Li, H. Z. \& Chen, J. Manipulating the pyruvate dehydrogenase bypass of a multi-vitamin auxotrophic yeast Torulopsis glabrata enhanced pyruvate production. Lett. Appl. Microbiol. 39, 199-206 (2004).

3. Yang, S. X., Wang, M., Wang, J., Liu, L. M. \& Chen, J. Optimization of Pyruvate Production Conditions Based on Environmental Adaptability of Torulopsis glabrata in a Pilot Plant Scale. Chin. J. Proc. Eng. 11, 1044-1049 (2011).

4. Causey, T., Shanmugam, K., Yomano, L. \& Ingram, L. Engineering Escherichia coli for efficient conversion of glucose to pyruvate. Proc. Natl. Acad. Sci. USA 101, 2235-2240 (2004).

5. Barrett, D. G. \& Yousaf, M. N. Poly(triol $\alpha$-ketoglutarate) as Biodegradable, Chemoselective, and Mechanically Tunable Elastomers. Macromolecules 41, 6347-6352, (2008).

6. Zhang, D. D. et al. Enhancement of $\alpha$-ketoglutarate production in Torulopsis glabrata: Redistribution of carbon flux from pyruvate to $\alpha$-ketoglutarate. Biotechnol. Bioprocess Eng. 14, 134-139 (2009).

7. Chen, X., Zhu, P. \& Liu, L. Modular optimization of multi-gene pathways for fumarate production. Metab. Eng. 33, 76-85 (2015).

8. Li, S., Xu, N., Liu, L. \& Chen, J. Engineering of carboligase activity reaction in Candida glabrata for acetoin production. Metab. Eng. 22, 32-39 (2014).

9. Chen, X. et al. Metabolic engineering of Torulopsis glabrata for malate production. Metab. Eng. 19, 10-16 (2013).

10. Gao, X., Xu, N., Li, S. \& Liu, L. Metabolic engineering of Candida glabrata for diacetyl production. PLoS One 9, e89854 (2014).

11. Dujon, B. et al. Genome evolution in yeasts. Nature 430, 35-44 (2004).

12. Borg-von Zepelin, M. et al. Epidemiology and antifungal susceptibilities of Candida spp. to six antifungal agents: results from a surveillance study on fungaemia in Germany from July 2004 to August 2005. J. Antimicrob. Chemother. 60, 424-428, (2007).

13. Roetzer, A., Gabaldon, T. \& Schuller, C. From Saccharomyces cerevisiae to Candida glabrata in a few easy steps: important adaptations for an opportunistic pathogen. FEMS Microbiol. Lett. 314, 1-9 (2011).

14. Cormack, B. P., Ghori, N. \& Falkow, S. An adhesin of the yeast pathogen Candida glabrata mediating adherence to human epithelial cells. Science 285, 578-582 (1999).

15. Bairwa, G., Rasheed, M., Taigwal, R., Sahoo, R. \& Kaur, R. GPI (glycosylphosphatidylinositol)-linked aspartyl proteases regulate vacuole homoeostasis in Candida glabrata. Biochem. J. 458, 323-334 (2014).

16. Miyata, R. \& Yonehara, T. Improvement of fermentative production of pyruvate from glucose by Torulopsis glabrata IFO 0005. J. Fermen. Bioeng. 82, 475-479 (1996).

17. Xu, N. et al. Reconstruction and analysis of the genome-scale metabolic network of Candida glabrata. Mol. Biosyst. 9, 205-216 (2013).

18. Li, Y., Chen, J., Lun, S. \& Rui, X. The important role of vitamins in the over-production of pyruvic acid. Acta. Microbiol. Sinica. 40, 528-534 (2000)

19. Liu, L., Yin, L., Li, H. \& Jian, C. Significant increase of glycolytic flux in Torulopsis glabrata by inhibition of oxidative phosphorylation. FEMS Yeast Res. 6, 1117-1129 (2006).

20. Zhou, J., Huang, L., Liu, L. \& Chen, J. Enhancement of pyruvate productivity by inducible expression of a F0F1-ATPase inhibitor INH1 in Torulopsis glabrata CCTCC M202019. J. Biotechnol. 144, 120-126 (2009).

21. Chaudhuri, R., Ansari, F. A., Raghunandanan, M. V. \& Ramachandran, S. FungalRV: adhesin prediction and immunoinformatics portal for human fungal pathogens. BMC Genomics 12, 192 (2011).

22. de Groot, P. W. J. et al. The Cell Wall of the Human Pathogen Candida glabrata: Differential Incorporation of Novel Adhesin-Like Wall Proteins. Eukaryot. Cell 7, 1951-1964 (2008).

23. Verstrepen, K. J. \& Klis, F. M. Flocculation, adhesion and biofilm formation in yeasts. Mol. Microbiol. 60, 5-15 (2006).

24. de Groot, P. W. J., Bader, O., de Boer, A. D., Weig, M. \& Chauhan, N. Adhesins in Human Fungal Pathogens: Glue with Plenty of Stick. Eukaryot. Cell 12, 470-481 (2013).

25. Guo, B., Styles, C. A., Feng, Q. \& Fink, G. R. A Saccharomyces gene family involved in invasive growth, cell-cell adhesion, and mating. Proc. Natl. Acad. Sci. USA 97, 12158-12163 (2000). 
26. Desai, C., Mavrianos, J. \& Chauhan, N. Candida glabrata Pwp7p and Aedlp are required for adherence to human endothelial cells. FEMS Yeast Res. 11, 595-601 (2011).

27. Verstrepen, K. J., Jansen, A., Lewitter, F. \& Fink, G. R. Intragenic tandem repeats generate functional variability. Nat. Genet. 37, 986-990 (2005).

28. Luo, G. \& Samaranayake, L. P. Candida glabrata, an emerging fungal pathogen, exhibits superior relative cell surface hydrophobicity and adhesion to denture acrylic surfaces compared with Candida albicans. APMIS 110, 601-610 (2002).

29. Patel, R. K. \& Jain, M. NGS QC Toolkit: a toolkit for quality control of next generation sequencing data. PLoS One 7, e30619 (2012).

30. Zerbino, D. R. \& Birney, E. Velvet: Algorithms for de novo short read assembly using de Bruijn graphs. Genome Res. 18, 821-829 (2008).

31. Boetzer, M., Henkel, C. V., Jansen, H. J., Butler, D. \& Pirovano, W. Scaffolding pre-assembled contigs using SSPACE. Bioinformatics 27, 578-579 (2011)

32. Nadalin, F., Vezzi, F. \& Policriti, A. GapFiller: a de novo assembly approach to fill the gap within paired reads. BMC Bioinformatics 13 Suppl 14, S8 (2012).

33. Tarailo-Graovac, M. \& Chen, N. Using RepeatMasker to identify repetitive elements in genomic sequences. Curr. Protoc. Bioinformatics, 4.10, 11-14 (2009).

34. Slater, G. S. \& Birney, E. Automated generation of heuristics for biological sequence comparison. BMC Bioinformatics 6, 31 (2005).

35. Keller, O., Kollmar, M., Stanke, M. \& Waack, S. A novel hybrid gene prediction method employing protein multiple sequence alignments. Bioinformatics 27, 757-763 (2011).

36. Koonin, E. V. et al. A comprehensive evolutionary classification of proteins encoded in complete eukaryotic genomes. Genome Biol. 5, R7 (2004).

37. Moriya, Y., Itoh, M., Okuda, S., Yoshizawa, A. C. \& Kanehisa, M. KAAS: an automatic genome annotation and pathway reconstruction server. Nucleic Acids Res. 35, W182-W185 (2007).

38. Pillai, L. et al. Developing a biocuration workflow for AgBase, a non-model organism database. Database (Oxford) 2012, bas038, (2012).

39. Lowe, T. M. \& Eddy, S. R. tRNAscan-SE: a program for improved detection of transfer RNA genes in genomic sequence. Nucleic Acids Res. 25, 955-964 (1997).

40. Lagesen, K. et al. RNAmmer: consistent and rapid annotation of ribosomal RNA genes. Nucleic Acids Res. 35, 3100-3108 (2007).

41. Altschul, S. F., Gish, W., Miller, W., Myers, E. W. \& Lipman, D. J. Basic local alignment search tool. J. Mol. Biol. 215, 403-410 (1990).

42. Langmead, B. \& Salzberg, S. L. Fast gapped-read alignment with Bowtie 2. Nat Methods 9, 357-359 (2012).

43. Li, H. et al. The Sequence Alignment/Map format and SAMtools. Bioinformatics 25, 2078-2079 (2009).

44. McKenna, A. et al. The Genome Analysis Toolkit: a MapReduce framework for analyzing next-generation DNA sequencing data. Genome Res. 20, 1297-1303 (2010).

45. Doran, A. G. \& Creevey, C. J. Snpdat: easy and rapid annotation of results from de novo snp discovery projects for model and nonmodel organisms. BMC Bioinformatics 14, 45 (2013).

46. Rutherford, K. et al. Artemis: sequence visualization and annotation. Bioinformatics 16, 944-945 (2000).

47. Liu, L. M., Li, Y., Li, H. Z. \& Chen, J. Manipulating the pyruvate dehydrogenase bypass of a multi-vitamin auxotrophic yeast Torulopsis glabrata enhanced pyruvate production. Lett. Appl. Microbiol. 39, 199-206 (2004).

48. Liu, L. M., Li, Y., Li, H. Z. \& Chen, J. Manipulating the pyruvate dehydrogenase bypass of a multi-vitamin auxotrophic yeast Torulopsis glabrata enhanced pyruvate production. Lett. Appl. Microbiol. 39, 199-206 (2004).

49. Liu, L., Li, Y., Zhu, Y., Du, G. \& Chen, J. Redistribution of carbon flux in Torulopsis glabrata by altering vitamin and calcium level. Metab. Eng. 9, 21-29 (2007).

50. Martin, F. P. et al. A top-down systems biology view of microbiome-mammalian metabolic interactions in a mouse model. Mol. Syst. Biol. 3, 112 (2007).

51. Folger, O. et al. Predicting selective drug targets in cancer through metabolic networks. Mol. Syst. Biol. 7 (2011).

52. McCloskey, D., Palsson, B. O. \& Feist, A. M. Basic and applied uses of genome-scale metabolic network reconstructions of Escherichia coli. Mol. Syst. Biol. 9, 661 (2013).

53. Verstrepen, K. J. \& Klis, F. M. Flocculation, adhesion and biofilm formation in yeasts. Mol. Microbiol. 60, 5-15 (2006).

54. Ramana, J. \& Gupta, D. FaaPred: a SVM-based prediction method for fungal adhesins and adhesin-like proteins. PLoS One 5, e9695 (2010).

55. Schultz, J., Milpetz, F., Bork, P. \& Ponting, C. P. SMART, a simple modular architecture research tool: identification of signaling domains. Proc. Natl. Acad. Sci. USA 95, 5857-5864 (1998).

56. Pierleoni, A., Martelli, P. L. \& Casadio, R. PredGPI: a GPI-anchor predictor. BMC Bioinformatics 9, 392, (2008).

57. Hall, T. A. BioEdit: a user-friendly biological sequence alignment editor and analysis program for Windows 95/98/NT. Nucleic Acids Symp. Ser. 41, 95-98 (1999).

\section{Acknowledgements}

This work was supported by the National High Technology Research and Development Program of China (2014AA021501), the Fundamental Research Funds of the Central Universities (JUDCF13009), the National Natural Science Foundation of China (21422602), and the Graduate Student Creative Research Programme of Jiangsu Province in 2013 (CXZZ13_0755).

\section{Author Contributions}

L.L. and N.X. conceived the project. N.X., J.L. and X.C. did fermentation experiments. N.X. and C.Y. completed genome sequencing and bioinformatics analyses. N.X. and X.C. validated cell virulence. N.X., L.L. and J.C. wrote the manuscript.

\section{Additional Information}

Accession Codes: The assembled C. glabrata CCTCC M202019 genome has been deposited at DDBJ/ENA/ GenBank under the accession AYJS00000000. The version described in this paper is version AYJS02000000.

Supplementary information accompanies this paper at http://www.nature.com/srep

Competing financial interests: The authors declare no competing financial interests.

How to cite this article: $\mathrm{Xu}, \mathrm{N}$. et al. Genome Sequencing of the Pyruvate-producing Strain Candida glabrata CCTCC M202019 and Genomic Comparison with Strain CBS138. Sci. Rep. 6, 34893; doi: 10.1038/srep34893 (2016). 
(c) (i) This work is licensed under a Creative Commons Attribution 4.0 International License. The images or other third party material in this article are included in the article's Creative Commons license, unless indicated otherwise in the credit line; if the material is not included under the Creative Commons license, users will need to obtain permission from the license holder to reproduce the material. To view a copy of this license, visit http://creativecommons.org/licenses/by/4.0/

(C) The Author(s) 2016 